\title{
BNREL
}

\section{Evaluating the Impacts of Battery Energy Storage System Functionalities on Distribution Systems Using Power Hardware-in-the-Loop Simulation}

Annabelle Pratt ${ }^{1}$, Jing Wang ${ }^{1}$, Nathan Ainsworth ${ }^{2}$, Kumaraguru Prabakar ${ }^{1}$, Murali Baggu ${ }^{1}$, Tom Bialek ${ }^{3}$, and Martha Symko-Davies

${ }^{1}$ Power Systems Engineering Center, National Renewable Energy Laboratory, Golden, Colorado, USA

${ }^{2}$ Nhu Energy, Inc., Tallahassee, Florida, USA

${ }^{3}$ San Diego Gas \& Electric Company, San Diego, California, USA

\section{Abstract}

A battery energy storage system (BESS) can provide various grid support services, including voltage regulation, peak shaving, and photovoltaic (PV) smoothing. This paper presents results from a power hardware-inthe-loop (PHIL) simulation that was performed to test and demonstrate the impacts of BESS functionalities on a distribution feeder.

The PHIL platform includes a simulated distribution grid in a real-time digital simulator (RTDS), AC and DC power amplifiers, and a battery inverter as the device under test. Guidelines on how to set up a PHIL platform to evaluate the impact of grid integrated systems are provided. Accelerated time-series PHIL simulations of peak shaving and volt-watt functionalities were performed using a 540-kVA BESS. These experimental results illustrate how PHIL simulations can be used to evaluate the impact of BESS functionalities on the distribution grid prior to installation in the field.

\section{Distribution Grid with BESS}

We based our evaluation on a San Diego Gas \& Electric Company (SDG\&E) feeder in which a lithium-ion-based BESS has been installed. Our research was encouraged by SDG\&E in light of their need to comply with the California Public Utilities Commission's requirement to meet a target of $165 \mathrm{MW}$ of cost-effective grid storage by year 2020

The simulated distribution feeder is a reduced-order representation of the full network. The full SDG\&E 2,174-node feeder model was reduced to an equivalent 7-bus model. It is a $12-\mathrm{kV}$ feeder with a peak load of $7.5 \mathrm{MW}$. The loads are modeled as dynamic loads. Two capacitor banks, both rated at $1.2 \mathrm{MVar}$, are used to regulate the voltage.

A 1.2-MW PV system is connected at the end of the feeder, and a 1$\mathrm{MW} / 3-\mathrm{MWh}$ BESS is connected upstream of the PV system. The loads are modeled as dynamic power sinks, and they are controlled by multiplying a per-unit load profile by a fixed load.

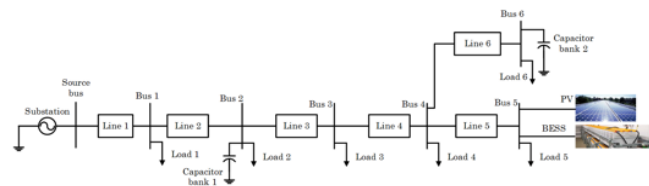

\section{Hardware-in-the-Loop Platform}

Prior to the installation of a BESS in the field, proper evaluation must be performed to understand how the BESS can impact the grid. The most popular hardware testing approach is hardware-in-the-loop testing, which has several benefits, such as repeatability, relatively low cost, and reliable results. PHIL combines the advantages of a complete numerical simulation method and actual hardware, and PHIL testing allows hardware devices to be evaluated in an environment that emulates various realistic field conditions.

The distribution feeder was simulated in an RTDS with a time step of 50 us. The PV system is modeled as a controlled current source. The input to the PV model specifies the output active power of the PV system, an the $\mathrm{PV}$ controller regulates the current to match the PV output active power to this input. We implemented an augmented ideal transformer PHIL interface method, and we present the design of the PHIL interface compensation algorithm to ensure stable and accurate PHIL simulation.

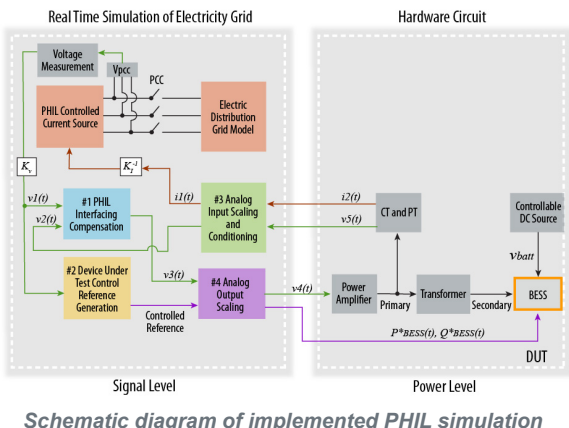

Schematic diagram of implemented PHIL simulation

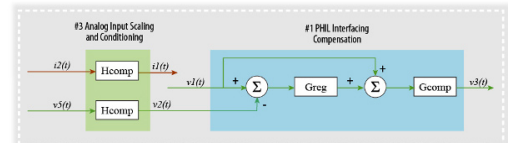

PHIL loop with the interface compensatio

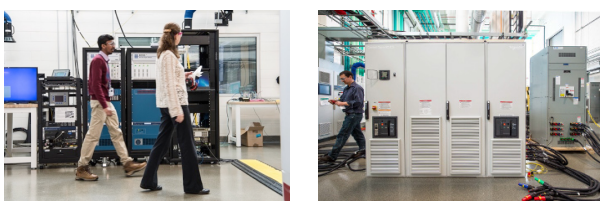

PHIL hardware in NREL's Energy Systems Integration Facility

\section{Results: Peak Shaving}

The peak day load and PV data sets were used. The BESS was inactive for the baseline test case, and it was operated to track a precalculated real power profile for the peak shaving test case. The BESS sinks real power (charging the battery) in the early morning near the minimum power, and it sources power (discharging) in the evening to reduce the peak. As a result, the peak source power is reduced.

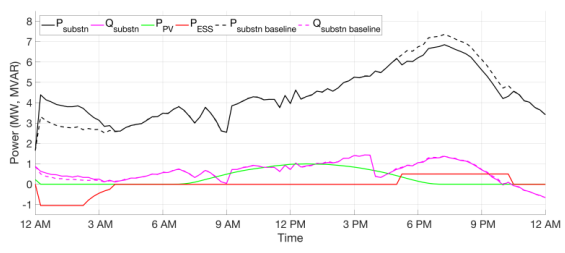

Feeder power profile results for peak load day with peak shaving

\section{Results: Volt-Watt}

The minimum day load and PV data set were tested with and without the BESS performing volt-watt compensation with no deadband. The BESS provides active power to compensate the morning and evening voltage dips. For this feeder, the BESS high rating to significantly impact the voltages.

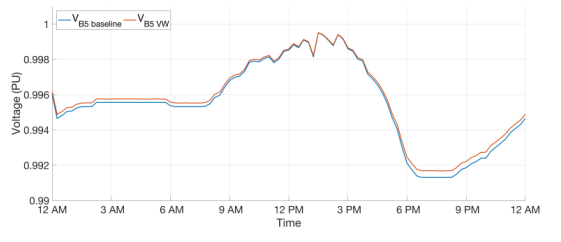

Comparison of voltage at BESS with and without volt-watt

\section{Conclusions}

Two functionalities-peak shaving and volt-watt-are simulated, and the experimental results are presented. These results demonstrate how a PHIL simulation platform can provide a valuable evaluation prior to field installation. Information from the PHIL simulations can be used by utility operators to determine how to operate their utility assets most effectively under different scenarios in the field presented. 